\title{
Secretion of N-ERC/mesothelin and expression of C-ERC/mesothelin in human pancreatic ductal carcinoma
}

\author{
KOICHI INAMI ${ }^{1,2}$, KAZUNORI KAJINO $^{1}$, MASAAKI ABE ${ }^{1}$, YOSHIAKI HAGIWARA ${ }^{1,3}$, \\ MASAHIRO MAEDA $^{3}$, MASAFUMI SUYAMA ${ }^{2}$, SUMIO WATANABE ${ }^{2}$ and OKIO HINO ${ }^{1}$
}

Departments of ${ }^{1}$ Pathology and Oncology and ${ }^{2}$ Gastroenterology, Juntendo University School of Medicine, 2-1-1 Hongo, Bunkyo-ku, Tokyo 113-8421; Immuno-Biological Laboratories, 5-1 Aramachi, Takasaki-shi, Gunma 370-0831, Japan

Received July 28, 2008; Accepted September 12, 2008

DOI: 10.3892/or_00000155

\begin{abstract}
ERC/mesothelin gene (MSLN) encodes a precursor protein, which is cleaved by proteases to generate $\mathrm{N}$-ERC/mesothelin and C-ERC/mesothelin. N-ERC/ mesothelin is a soluble protein, also known as megakaryocytepotentiating factor, which is released into extracellular space. N-ERC/mesothelin is known to be a serum marker of mesothelioma. We have previously developed an enzymelinked immunosorbent assay system for $\mathrm{N}-\mathrm{ERC} / \mathrm{mes}$ thelin, which can detect mesothelioma. C-ERC/mesothelin is expressed in normal mesothelial cell, pancreatic cancers, ovarian cancers, mesotheliomas and some other cancers. Pancreatic ductal carcinoma remains a fatal disease because its diagnosis often occurs very late. In this study, we examined ERC/mesothelin expression in human pancreatic cancer cell lines (MIA-PaCa2, PK-1, KP-3, TCC-PAN2, PK-59 and PK$45 \mathrm{H})$ by reverse transcription-polymerase chain reaction and immunoblotting and $\mathrm{N}-\mathrm{ERC} /$ mesothelin concentration in the supernatant of cultured cancer cells by the ELISA system. We also investigated C-ERC/mesothlein expression in human pancreatic ductal carcinoma tissues by immunostaining using 5B2 anti-mesothelin monoclonal antibody and $\mathrm{N}-\mathrm{ERC} / \mathrm{mes}$ othelin concentration in sera obtained from patients with pancreatic ductal carcinoma via ELISA. In vitro, $\mathrm{N}-\mathrm{ERC} / \mathrm{mesothelin}$ concentration in cell culture medium nearly correlated with the expression level of $\mathrm{C}$ $\mathrm{ERC} /$ mesothelin. Although C-ERC/mesothelin was frequently expressed in human pancreatic ductal carcinoma, serum N$\mathrm{ERC} / \mathrm{mes}$ thelin concentration of cancer patients was equivalent to healthy controls. N-ERC/mesothelin was not useful as a serum marker of pancreatic ductal carcinoma, but because of frequent expression, C-ERC/mesothelin might be useful as a target of molecular imaging and immunotherapy.
\end{abstract}

Correspondence to: Dr Okio Hino, Department of Pathology and Oncology, Juntendo University School of Medicine, 2-1-1 Hongo, Bunkyo-ku, Tokyo 113-8421, Japan

E-mail: ohino@med.juntendo.ac.jp

Key words: ERC/mesothelin, pancreatic ductal carcinoma, ELISA, immunohistochemistry

\section{Introduction}

ERC/mesothelin gene (MSLN) encodes a $71 \mathrm{kDa}$ precursor protein, which is cleaved by proteases to yield $31 \mathrm{kDa} \mathrm{N}$ terminal (N-ERC/mesothelin) and $40 \mathrm{kDa}$ C-terminal (C-ERC/ mesothelin) proteins $(1,2)$. N-ERC/mesothelin, originally identified as megakaryocyte-potentiating factor (MPF), is soluble and released into extracellular space (1-9). C-ERC/ mesothelin is a glycoprotein tethered to the cell surface by glycosyl-phosphatidyl-inositol (GPI) anchor. Some forms of C-ERC/mesothelin are released into extra-cellular space by aberrant splicing or proteases $(1,2,10-13)$.

$\mathrm{N}-\mathrm{ERC} / \mathrm{mes}$ thelin/MPF was isolated from the medium of cultured pancreatic cancer cells $(3,4)$ and is known to be a serum marker of mesothelioma (5-9). C-ERC/mesothelin is expressed not only in normal mesothelial cells of the pleura, pericardium and peritoneum, but also in malignant cells of pancreatic ductal carcinomas, ovarian cancers, mesotheliomas and some other cancers $(1,14-17)$. C-ERC/ mesothelin can be detected in the sera of patients with ovarian carcinoma and mesothelioma $(10-12,18)$. Previously, we discovered Erc, which is expressed in renal cell cancers of Eker rats. We also confirmed that Erc is a homolog of human MSLN (19-21).

Pancreatic ductal carcinoma remains a fatal disease because of its poor prognosis. Unfortunately, the diagnosis of pancreatic ductal carcinoma often occurs very late and consequently, $<40 \%$ of patients are candidates for tumor resection (22-24). Thus, the overall 5-year survival rate of these patients is $<10 \%(23,24)$. On the other hand, those of patients with stage I disease is $58.1 \%$ (25). Novel strategy for early diagnosis of pancreatic ductal carcinoma is warranted.

ERC/mesothelin is expressed in human pancreatic ductal carcinoma and not expressed in normal pancreatic tissue $(15,16)$. Previous studies showed the usefulness of N-ERC/ mesothelin and C-ERC/mesothelin as diagnostic markers for C-ERC/mesothelin expressing tumors (5-12). To date, there has been no report about the effectiveness of N-ERC/ Meosthelin and $\mathrm{C}$-ERC/mesothelin as serum markers of pancreatic ductal carcinoma. We have previously devised a novel enzyme-linked immunosorbent assay (ELISA) system for $\mathrm{N}-\mathrm{ERC} / \mathrm{mes}$ thelin and showed that it is useful for diagnosis of human mesothelioma (5-7). In this study, we 
examined the expression of $\mathrm{C}$ - and $\mathrm{N}-\mathrm{ERC} / \mathrm{mes}$ thelin in cultured pancreatic cancer cell lines and human pancreatic ductal carcinomas and investigated the usefulness of our ELISA system as a diagnostic procedure of human pancreatic ductal carcinoma.

In the cultured cells, the concentration of N-ERC/ mesothelin in the medium nearly correlated with the expression of $\mathrm{C}$-ERC/mesothelin. C-ERC/mesothelin was frequently expressed in human pancreatic ductal carcinoma. There was, however, no increase in $\mathrm{N}$-ERC/mesothelin concentration in the sera of pancreatic cancer patients compared with that of normal controls. Although N-ERC/ mesothelin is established as a reliable marker for mesothelioma, N-ERC/mesothelin is not useful as a diagnostic marker of pancreatic ductal carcinoma. As for C-ERC/ mesothelin, it might be useful as a target of molecular imaging and immunotherapy, because of its frequent expression.

\section{Materials and methods}

Pancreatic cancer cell lines. MIA-PaCa2 and PK-1 were provided by Cell Resource Center for Biomedical Research, Tohoku University, Sendai-shi, Miyagi, Japan. KP-3 and TCC-PAN2 were provided by Health Science Research Resources Bank, Sennan-shi, Osaka, Japan. PK-59 and PK$45 \mathrm{H}$ were provided by RIKEN CELL BANK, Tsukuba-shi, Ibaraki, Japan. MIA-PaCa2 was cultured in Dulbecco's modified Eagle's medium supplemented with $10 \%$ fetal calf serum, $100 \mathrm{U} / \mathrm{ml}$ penicillin and $0.1 \mathrm{mg} / \mathrm{ml}$ streptomycin. PK-1, KP-3, TCC-PAN2, PK-59 and PK-45H were cultured in RPMI-1640 medium supplemented with $10 \%$ fetal calf serum, $100 \mathrm{U} / \mathrm{ml}$ penicillin and $0.1 \mathrm{mg} / \mathrm{ml}$ streptomycin. Culture supernatants and cells were harvested $48 \mathrm{~h}$ after culturing at $37^{\circ} \mathrm{C}$ and $5 \% \mathrm{CO}_{2}$ atmosphere, upon reaching $>80 \%$ confluency.

Reverse transcription-polymerase chain reaction (RT-PCR). mRNA levels of ERC/mesothelin in the cultured cells (MIA$\mathrm{PaCa} 2, \mathrm{PK}-1, \mathrm{KP}-3$, TCC-PAN2, PK-59 and PK-45H) were analyzed by RT-PCR. Cells in petri dishes were lysed by the acid guanidinium thiocyanate-phenol-chloroform extraction method (26) using TRIzol reagent (Invitrogen, Carlsbad, CA, USA). Total RNAs were extracted from these lysates following manufacturer's instructions. Total RNA $(1 \mu \mathrm{g})$ was reverse transcribed for $30 \mathrm{~min}$ at $50^{\circ} \mathrm{C}$ and subjected to polymerase chain reaction amplification. The primers used to amplify the ERC/mesothelin were: sense 5'-CAAGAA GTGGGAGCTGGAAG-3' and antisense 5'-GTCTCCAGG GACGTCACATT-3'. As a control for RT-PCR, B-actin mRNA was amplified using the following $B$-actin-specific primers: sense 5'-CCGCGAGAAGATGACCCAGA-3'; and antisense 5'-CAGGAGGAGCAATGATCTTG-3'. All primers were purchased from Operon (Tokyo, Japan). RTPCR was carried out in an MBS Satellite 0.2 (Thermo Fisher Scientific, Kanagawa, Japan), using Titan RT-PCR System (Roche Diagnostics GmbH, Mannheim, Germany) following manufacturer's instructions. After an initial denaturation step of 4 min at $94^{\circ} \mathrm{C}$, each sample was subjected to 25 cycles of amplification (denaturation, $30 \mathrm{sec}$ at $94^{\circ} \mathrm{C}$; annealing, $30 \mathrm{sec}$ at $50^{\circ} \mathrm{C}$; and elongation, $1 \mathrm{~min}$ at $68^{\circ} \mathrm{C}$ ) followed by a final elongation step for $10 \mathrm{~min}$ at $68^{\circ} \mathrm{C}$. PCR product $(10 \mu \mathrm{l})$ was analyzed on a $2 \%$ agarose gel containing $0.5 \mu \mathrm{g} / \mathrm{ml}$ ethidium bromide.

Immunoblotting. MIA-PaCa2, PK-1, KP-3, TCC-PAN2, PK-59 and PK-45H cells in petri dishes were lysed in a solution containing $2 \%$ sodium dodecylsulfate, $10 \%$ glycerol, $50 \mathrm{mM}$ Tris-HCl $(\mathrm{pH}$ 6.8) and $100 \mathrm{mM}$ dithiothreitol, followed by boiling for $2 \mathrm{~min}$. These lysates were electrophoresed in $10 \%$ Laemmli gels and transferred onto nitrocellulose membranes. Membranes were blocked in $1 \%$ skim milk in phosphate-buffered saline with $0.1 \%$ Tween-20 (PBS-T) for $1 \mathrm{~h}$ at room temperature. Next, membranes were incubated with 5B2 anti mesothelin antibody (Novocastra Laboratory Vision BioSystems, Boston, MA, USA, 1:100 dilution) or AC15 anti B-actin antibody (Sigma, St. Louis, MO, USA, 1:5000 dilution) in PBS-T with $1 \%$ skim milk for $1 \mathrm{~h}$ at room temperature. EnVision+ system labeled polymer-horseradish peroxidase (HRP) (K4000 or K4001 purchased from Dako, Glostrup, Denmark) at a 100 -fold dilution in PBS-T with $1 \%$ skim milk was added and allowed to react with the membrane at room temperature for $1 \mathrm{~h}$. ECL detection system (GE Healthcare, Buckinghamshire, UK) was used to visualize ERC/mesothelin on the membrane.

Human subjects. Patients with ductal pancreatic carcinoma, treated in Juntendo hospital between April 1, 2006 and November 30, 2007, were evaluated in this study. Pathological diagnosis was based on the histological analysis of tissue samples obtained from pancrearectomy or endoscopic ultrasonography guided fine needle aspiration biopsy (FNA), using sterile 21-gauge needles. This study was approved by the Institutional Review Board of Juntendo University School of Medicine and its hospital. Patients gave their signed informed consent. Nineteen healthy controls were sampled at random from a database, as described (6), with an age range of 50-79 years.

Immunohistochemistry. Tissue sections, $3 \mu \mathrm{m}$ thick, were prepared from archival formalin-fixed, paraffin-embedded specimens. After deparaffinization, the tissue sections were heated in $10 \mathrm{mM}$ citrate buffer $(\mathrm{pH}$ 6.0) for antigen retrieval and then treated with $3 \%$ hydrogen peroxide. Next, the sections were incubated with primary antibody solutions diluted in Tris-buffered saline with $0.1 \%$ Tween-20 (TBS-T) overnight at $4^{\circ} \mathrm{C}$. We used mouse monoclonal anti human C-ERC/mesothelin antibody 5B2 (1:50 dilution) as the primary antibody and EnVision+ system labeled with polymer-HRP (Dako) as the secondary antibody. Diaminobenzidine was used as the substrate for peroxidase. For immunostained slides, the intensity of staining was semiquantitatively graded on a scale of $1+$ to $3+$ and the proportion of stained ducts of cancer gland was graded as $0 \%, 1$ to $<10 \%, 10-50 \%$ and $>50 \%$.

ELISA. N-ERC/mesothelin concentration in sera and cell culture supernatants (MIA-PaCa2, PK-1, KP-3, TCC-PAN2, PK-59 and PK-45H) were analyzed by sandwich ELISA method. Sandwich ELISA method was performed as 
A

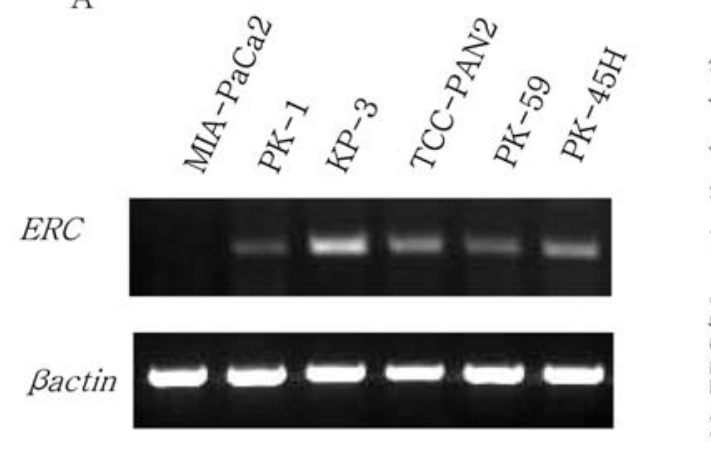

B
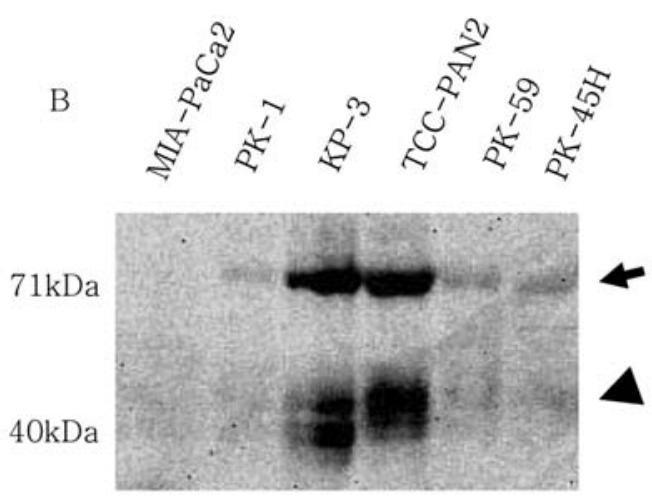

Bactin
$\mathrm{C}$

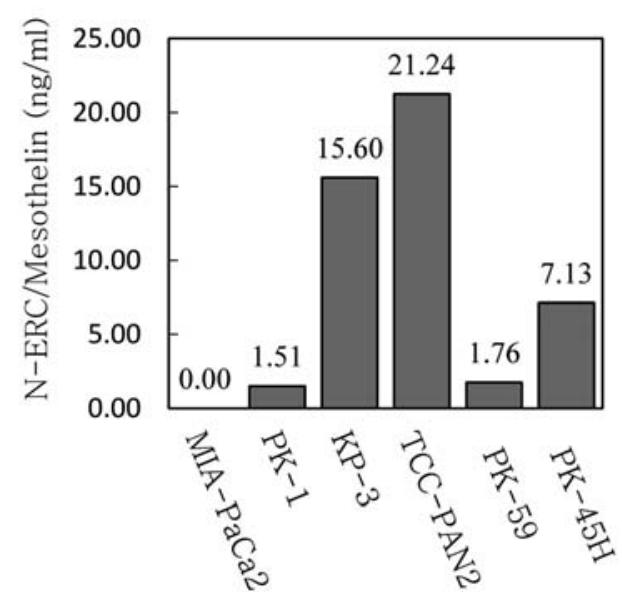

Figure 1. ERC/mesothelin expression in human pancreatic cancer cell lines. (A) ERC/mesothelin transcript in human pancreatic cancer cell lines detected by RT-PCR. (B) ERC/mesothelin protein in human pancreatic cancer cell lines detected by immunoblotting. Arrow, precursor ERC/mesothelin (71 kDa); Arrowhead, C-ERC/mesothelin (40 kDa). (C) Secreted N-ERC/mesothelin in the cell culture medium of human pancreatic cancer cell lines detected by ELISA.

previously described $(5,6)$, using $7 \mathrm{E} 7$ monoclonal antibody and HRP-conjugated polyclonal antibody-282. Absorbance at $450 \mathrm{~nm}$ was measured in an ELISA reader (E-MAX; Molecular Devices, Sunnyvale, CA, USA).

Statistical analysis. We analyzed ELISA data using JMP and SAS version 8.1.3 (SAS Institute, Cary, CA, USA). To compare serum concentration between groups, the MannWhitney test was used. $\mathrm{P}<0.05$ was considered statistically significant.

\section{Results}

ERC/mesothelin expression in human pancreatic cancer cell lines. RT-PCR revealed ERC/mesothelin mRNA expression in most of the investigated pancreatic cancer cell lines, except for MIA-PaCa2 (Fig. 1A). Immunoblotting showed strong ERC/mesothelin and $\mathrm{C}-\mathrm{ERC} / \mathrm{mes}$ thelin expression in 2 of 6 cell lines (KP-3 and TCC-PAN2) and weak expression in PK-1, PK-59 and PK-45H. MIA-PaCa2 did not demonstrate any ERC/mesothelin and C-ERC/ mesothelin expression (Fig. 1B). N-ERC/mesothelin was secreted into the culture supernatants of 5 cell lines, of which 2 (KP-3 and TCC-PAN2) showed high N-ERC/ mesothelin concentration. PK-1, PK-59 and PK-45H secreted
$\mathrm{N}-\mathrm{ERC} /$ mesothelin moderately. MIA-PaCa2 did not secrete $\mathrm{N}-\mathrm{ERC} / \mathrm{mes}$ thelin at all (Fig. 1C).

C-ERC/mesothlein expression in human pancreatic ductal carcinoma. Of 19 tissue samples, 8 were obtained from pancrearectomy and 11 from FNA. They included 10 men and 9 women with age range of 40-78 years (mean 69.8) and consisted of one stage I, 14 stage III and 4 stage IV patients (the International Union against Cancer classification). The immunostaining results are shown in Table I. Positive staining for C-ERC/mesothelin was seen in 14 of the 19 samples. Six of 11 samples from FNA and all of the samples from pancreatic resections showed positive staining. The staining pattern was often focal and cytoplasmic with polarity to apical membrane (Fig. 2A and B). In some cases, polarity of the signal was weak or none (Fig. 2C).

Serum N-ERC/mesothelin levels in patients with pancreatic ductal carcinoma. Serum samples from 19 patients were obtained before surgery, chemotherapy, or any other therapies. The sera were evaluated for $\mathrm{N}-\mathrm{ERC} / \mathrm{mes}$ thelin. The 19 age-matched healthy control samples, from the database as described (6), included 10 men and 9 women with age range of 50-79 years (mean 65 ). There was no significant difference in serum $\mathrm{N}-\mathrm{ERC} / \mathrm{mes}$ othelin 

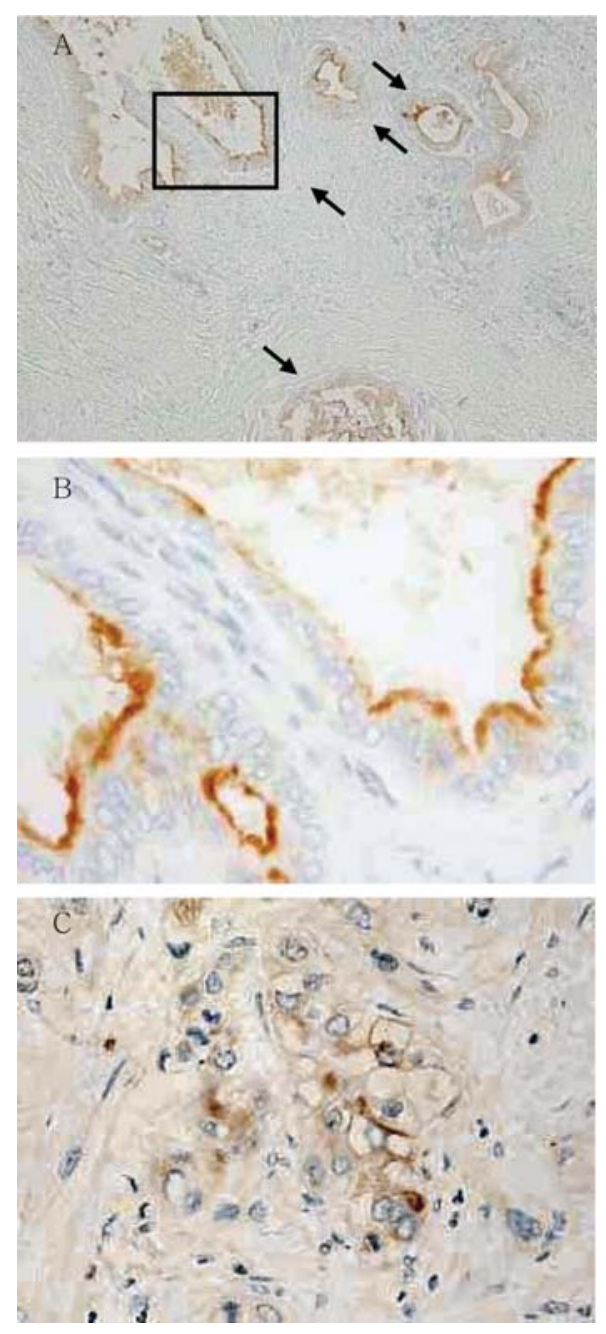

Figure 2. C-ERC/mesothelin expression in human pancreatic cancer tissue. (A) Immunohistochemical staining of C-ERC/mesothelin using 5B2 antibody, magnification (x40). Positive staining is shown by arrows. (B) Same sample as (A), respectively, with higher magnification (x200). An area covered by a square frame in (A) is expanded. (C) Another 5B2 stained sample with magnification (x200).
Table I. C-ERC/mesothelin immunostaining results of human pancreatic ductal carcinoma.

\begin{tabular}{lcccccccc}
\hline & \multicolumn{3}{c}{ Intensity } & & \multicolumn{4}{c}{ Proportion } \\
\cline { 2 - 4 } \cline { 6 - 8 } Sample & $1+$ & $2+$ & $3+$ & & $<$ & $<10$ & $10-50$ & $>50$ \\
\hline FNA & 3 & 1 & 2 & 5 & 3 & 1 & 2 \\
Operation & 2 & 4 & 2 & 0 & 1 & 4 & 3 \\
\hline Total & 5 & 5 & 4 & 5 & 4 & 5 & 5
\end{tabular}

The intensity of staining was semiquantitatively graded on a scale of $1+$ to $3+$ and the proportion of stained ducts of cancer gland was graded as $0 \%, 1$ to $<10 \%, 10-50 \%,>50 \%$.

concentration between cancer patients and the healthy control group ( $\mathrm{P}=0.569)$ (Fig. 3A). Between patients with resectable tumor and those with far advanced unresectable tumor, there was no significant difference in serum N-ERC/ mesothelin concentration ( $\mathrm{P}=0.710)$ (Fig. 3B).

\section{Discussion}

In the present study, we examined $\mathrm{C}$ - and $\mathrm{N}-\mathrm{ERC} /$ mesothelin expression in the two patients with pancreatic ductal carcinoma and cultured pancreatic cancer cell lines. The expression of C-ERC/mesothelin was studied by immunoblotting of cultured cell lysates or by immunohistochemical staining of carcinoma tissue. The concentration of $\mathrm{N}-\mathrm{ERC} /$ mesothelin in the supernatant of cultured cells or in sera of patients was measured by the ELISA system established by us (5-7). Our study indicated that N$\mathrm{ERC} /$ mesothelin concentration in supernatants correlated with the expression levels of C-ERC/mesothelin in cultured cells. Human pancreatic ductal carcinoma frequently expressed C-ERC/mesothelin. Contrary to our initial expectation, we did not find any significant difference in the

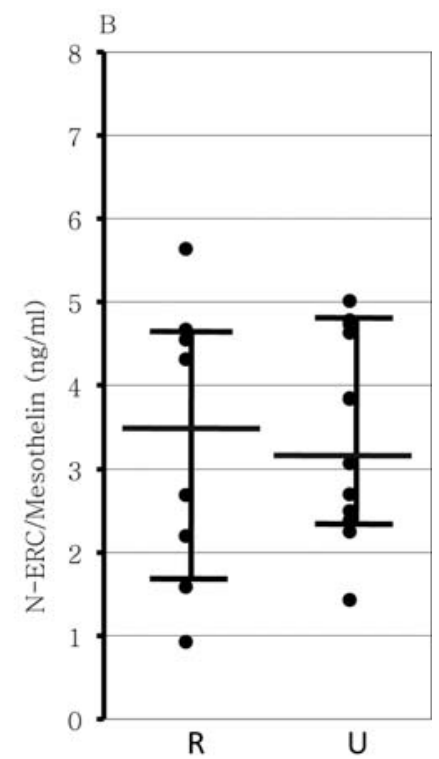

Figure 3. Scatter plots of serum N-ERC/mesothelin concentration. (A) Comparison of N-ERC/mesothelin concentration in sera from patients with pancreatic ductal carcinoma (P) and healthy controls (C). (B) Comparison of patients with resectable tumor (R) and unresectable far advanced tumor (U). 
serum concen-tration of N-ERC/mesothelin between patients with pancreatic ductal carcinoma and normal controls.

Almost all human pancreatic cancer cell lines, except for MIA-PaCa2, expressed mRNA of ERC/mesothelin. KP-3 and TCC-PAN2 strongly expressed C-ERC/mesothelin protein and secreted high amounts of $\mathrm{N}-\mathrm{ERC} / \mathrm{mes}$ thelin, while MIA-PaCa2 did not express $\mathrm{C}-\mathrm{ERC} / \mathrm{mes}$ othelin nor secrete $\mathrm{N}-\mathrm{ERC} / \mathrm{mes}$ thelin at all. N-ERC/mesothelin concentration in the supernatants of cultured cells almost correlated with C-ERC/mesothelin expression.

All surgically resected samples showed positive staining for C-ERC/mesothelin. However, five of 11 FNA cases showed negative staining. Baruch et al reported that $>50 \%$ of samples from FNA are negative for C-ERC/mesothelin because the staining pattern is most often focal and unevenly distributed (16). Argani et al reported that in tissue sections of pancreatic adenocarcinomas, diffuse staining was seen in only $30 \%$ of the mesothelin positive tumors (15). There is the possibility that our staining data of FNA samples, as shown in Table I, may have underestimated the actual expression of C-ERC/mesothelin because of the small and limited volume of FNA samples. Either way, we and others have shown that human pancreatic ductal carcinoma frequently expressed C-ERC/mesothelin. The staining pattern of pancreatic ductal carcinoma was cytoplasmic with or without polarity to apical membrane, while that of mesothelioma was membranous $(1,5,7,17,20)$.

Based on these results, we investigated whether the serum level of $\mathrm{N}-\mathrm{ERC} / \mathrm{mes}$ othelin could be a novel diagnostic marker of human pancreatic ductal carcinoma, using our previously reported ELISA system for detection (5-7). We age-matched the patients and healthy controls, because $\mathrm{N}-\mathrm{ERC} / \mathrm{mes}$ thelin in the sera has a tendency to elevate as people get older. Unexpectedly, we found that $\mathrm{N}-\mathrm{ERC} / \mathrm{mes}$ thelin in sera of patients with pancreatic ductal carcinoma was comparable to those of healthy controls.

Other ERC/mesothelin expressing tumors, including ovarian cancers and mesotheliomas, can be detected by measurement of serum C- or N-ERC/mesothelin concentration (5-12). We have shown that our ELISA system for N-ERC/ mesothelin also detects mesotheliomas (5-7). Presently, the reason why $\mathrm{N}-\mathrm{ERC} /$ mesothelin was not increased in the sera of patients with pancreatic ductal carcinoma while C-ERC/ mesothelin was frequently expressed in the carcinoma tissues is unknown. In the far advanced pancreatic ductal carcinoma patients with stage IV disease, N-ERC/mesothelin concentration was not higher than in the patients with stage I-III diseases (data not shown). Thus, it appears that the proportion of $\mathrm{C}$-ERC/mesothelin expressing ducts of the gland was not the reason. It was considered that differences between in vitro and in vivo conditions, for example changes in the micro-environments such as differential expression of proteases and their inhibitors, or tumor vascularity, may contribute to this discrepancy. High concentration of serum $\mathrm{N}$-ERC/mesothelin indicates a patient that does not have pancreatic ductal carcinoma, but other C-ERC/mesothelin expressing tumor, perhaps mesothelioma. In this case, examination for pancreatic ductal carcinoma could be omitted.
In conclusion, $\mathrm{N}-\mathrm{ERC} /$ mesothelin concentration in supernatants correlated with the expression levels of C-ERC/ mesothelin in cultured cells. Human pancreatic ductal carcinoma frequently expressed C-ERC/mesothelin. However, serum $\mathrm{N}-\mathrm{ERC} /$ mesothelin concentration of cancer patients was equivalent to healthy controls. N-ERC/mesothelin was not useful as a serum marker of pancreatic ductal carcinoma. Because of frequent expression of C-ERC/mesothelin in pancreatic ductal carcinoma tissues, there is a possibility that imaging detection system or immunotherapy, using C-ERC/ mesothelin, will be developed in the future.

\section{Acknowledgments}

We would like to thank, Masumi Maruo, Naoko Aoki, Kazu Shiomi, Danqing Zhang, Toshiyuki Kobayashi and members of the Department of Gastroenterology, Juntendo hospital, for their help in management of this study. This work is supported by a Grant-in-Aid for Cancer Research and Grants-in-Aid for Scientific Research from the Ministry of Education, Culture, Sports and Science and Technology of Japan and the Ministry of Health, Labor and Welfare of Japan. This study was partially supported by a consignment expense for Molecular Imaging Program on 'Research Base for PET Diagnosis' from Ministry of Education, Culture, Sport and Science and Technology, Government of Japan.

\section{References}

1. Chang K and Pastan I: Molecular cloning of mesothelin, a differentiation antigen present on mesothelium, mesotheliomas, and ovarian cancers. Proc Natl Acad Sci USA 93: 136-140, 1996.

2. Hassan R, Bera T and Pastan I: Mesothelin: a new target for immunotherapy. Clin Cancer Res 10: 3937-3942, 2004.

3. Yamaguchi N, Hattori K, Oh-eda M, Kojima T, Imai N and Ochi N: A novel cytokine exhibiting megakaryocyte potentiating activity from a human pancreatic tumor cell line HPC-Y5. J Biol Chem 269: 805-808, 1994.

4. Kojima T, Oh-eda M, Hattori K, et al: Molecular cloning and expression of megakaryocyte potentiating factor cDNA. J Biol Chem 270: 21984-21990, 1995.

5. Shiomi K, Miyamoto H, Segawa T, et al: Novel ELISA system for detection of N-ERC/mesothelin in the sera of mesothelioma patients. Cancer Sci 97: 928-932, 2006.

6. Shiomi K, Hagiwara Y, Sonoue K, et al: Sensitive and specific new enzyme-linked immunosorbent assay for N-ERC/Mesothelin increases its potential as a useful serum tumor marker for mesothelioma. Clin Cancer Res 14: 1431-1437, 2008.

7. Hino $\mathrm{O}$ and Shiomi K: Diagnostic biomarker of asbestos-related mesothelioma: Example of translational research. Cancer Sci 98: 1147-1157, 2007.

8. Scholler N, Fu N, Yang Y, et al: Soluble member(s) of the mesothelin/megakaryocyte potentiating factor family are detectable in sera from patients with ovarian carcinoma. Proc Natl Acad Sci USA 96: 11531-11536, 1999.

9. Onda M, Nagata S, Ho M, et al: Megakaryocyte potentiation factor cleaved from mesothelin precursor is a useful tumor marker in the serum of patients with mesothelioma. Clin Cancer Res 12: 4225-4231, 2006.

10. Hassan R, Remaley AT, Sampson ML, et al: Detection and quantitation of serum mesothelin, a tumor marker for patients with mesothelioma and ovarian cancer. Clin Cancer Res 12: 447-453, 2006.

11. Robinson BW, Creaney J, Lake R, et al: Mesothelin-family proteins and diagnosis of mesothelioma. Lancet 15: 1612-1616, 2003.

12. Robinson BW, Creaney J, Lake R, Nowak A, Musk AW, de Klerk N, Winzell P, Hellstrom KE and Hellstrom I: Soluble mesothelin-related protein - a blood test for mesothelioma. Lung Cancer 49: S109-S111, 2005. 
13. Sapede C, Gauvrit A, Barbieux I, et al: Aberrant splicing and protease involvement in mesothelin release from epithelioid mesothelioma cells. Cancer Sci 99: 590-594, 2008.

14. Chang K, Pastan I and Willingham M: Frequent expression of the tumor antigen CAK1 in squamous-cell carcinomas. Int $\mathbf{J}$ Cancer 51: 548-554, 1992.

15. Argani P, Iacobuzio-Donahue C, Ryu B, et al: Mesothelin is overexpressed in the vast majority of ductal adenocarinomas of the pancreas: identification of a new pancreatic cancer marker by serial analysis of gene expression (SAGE). Clin Cancer Res 7: 3862-3868, 2001

16. Baruch AC, Wang H, Staerkel GA, Evans DB, Hwang RF and Krishnamurthy S: Immunocytochemical study of the expression of mesothelin in fine-needle aspiration biopsy specimens of pancreatic adenocarcinoma. Diagn Cytopathol 35: 143-147, 2007.

17. Yaziji H, Battifora H, Barry TS, et al: Evaluation of 12 antibodies for distinguishing epithelioid mesothelioma from adenocarcinoma: identification of a three-antibody immunohistochemical panel with maximal sensitivity and specificity. Mod Pathol 19: 514-523, 2006.

18. Segawa T, Hagiwara Y, Ishikawa K, et al: MESOMARK kit detects C-ERC/mesothelin, but not SMRP with C-terminus. Biochem Biophys Res Commun 369: 915-918, 2008.
19. Yamashita Y, Yokoyama M, Kobayashi E, Takai S and Hino O: Mapping and determination of the cDNA sequence of the Erc gene preferentially expressed in renal cell carcinoma in the Tsc2 gene mutant (Eker) rat model. Biochem Biophys Res Commun 275: 134-140, 2000.

20. Maeda M and O Hino: Molecular tumor marker for asbestosrelated mesothelioma: serum diagnostic markers. Pathol Int 56: 649-654, 2006

21. Maeda M and O Hino: Blood test for asbestos-related mesothelioma. Oncology 71: 26-31, 2006.

22. Wray CJ, Ahmad SA, Matthews JB and Lowy AM: Surgery for pancreatic cancer: recent controversies and current practice. Gastroenterology 128: 1626-1641, 2005.

23. Li D, Xie K, Wolff R and Abbruzzese JL: Pancreatic cancer. Lancet 363: 1049-1057, 2004.

24. Matsuno S, Egawa S, Fukuyama S, et al: Pancreatic cancer registry in Japan: 20 years of experience. Pancreas 28: 219-230, 2004.

25. Egawa S, Takeda K, Fukuyama S, Motoi F, Sunamura M and Matsuno S: Clinicopathological aspects of small cancer. Pancreas 28: 235-240, 2004.

26. Chomczynski $P$ and Sacchi N: Single-step method of RNA isolation by acid guanidinium thiocyanate-phenol-chloroform extraction. Anal Biochem 162: 156-159, 1987. 